\title{
Parameter Identification of Human Body in Passive State Based on LMD Xin Jin ${ }^{1}$, Kedong Zhou ${ }^{1}$, Lei He ${ }^{1}$, Xueying Huang ${ }^{1}$ and Junbin Zhang $^{2}$ \\ ${ }^{1}$ School of Mechanical Engineering, Nanjing University of Science and Technology, Nanjing 210094, Jiangsu, China \\ ${ }^{2}$ Unit 63856 of PLA, Baicheng 137001, Jilin, China \\ ”inx0811@126.com
}

Keywords: parameter identification, human body, LMD

Abstract. In this paper, the physical parameters of human body in passive state are identified based on Local Mean Decomposition (LMD). The time-acceleration signal of human shoulder is gathered in the shooting test. LMD is used to obtain the Product Function (PF) components of the signal. The frequency-damping ratios are calculated at last based on the PF components. The comparison with existing parameters shows that the identification result is credible, and they are of great importance for the study of human biomechanics.

\section{Introduction}

The identification of modal parameter of structures is widely used in mechanical engineering, aerospace, civil, defense industry and other fields. In accordance with whether the system's stiffness, damping, or mass parameters will change with time, the system can be distinguished as time-varying and time-invariant system. There are many researches about modal parameter identification method for both systems, which are also more mature. However, the researches have not yet involved biodynamics, which is the development direction of mechanical engineering and similar fields.

The human body is a complicated system. According to the biology research [1], the reaction time of the nervous system is about $150 \sim 200 \mathrm{~ms}$, while the reaction time of muscle is above $350 \mathrm{~ms}$. Take a weapon with firing rate of 600 rounds per minute [2] for example: while shooting rounds are less than three, the shooter is still in passive state, the muscle is not energized. During this period, the stiffness and damping of human body are considered to be constant. However, when shooting rounds are over three, the shooter is in active state, and the muscle is energized. During this period, the stiffness and damping of human body are considered to be changed.

In this paper, a shooting test of one round is analyzed for two reasons: 1) the human body is in passive state, so the stiffness and damping are constant; 2) the force duration is very short, so the human body can be considered as free vibration. The time-acceleration signal of human shoulder is gathered in the test. Then, LMD is used to process the signal, and the PF components are obtained. The Modal Natural Frequency $f_{i}$ and parameter $n_{i}$ are calculated by liner fitting the instantaneous frequency and instantaneous amplitude (after natural logarithm). The Frequency-Damping Ratios are calculated at last by dividing $f_{i}$ by $n_{i}$. Results in reference [3] show that the identification result is credible.

\section{Parameter Identification Method}

\section{A. LMD}

The Local Mean Decomposition (refers to LMD) can self-adaptively divide a multi-component signal into several PF (Product Function, refers to PF) components with physical significance [4]. Each PF component is the product of a pure FM signal and an envelope signal, and the instantaneous frequency of the component is directly educed from pure FM signal, and the envelope signal is the amplitude. The original signal and its time and frequency distribution can be obtained by combining the instantaneous frequency and amplitude of all components. Essentially, the LMD method means dividing the multi-component signal into frequency-modulated and amplitude-modulated signals. 
The essence of LMD method is a stepwise decomposition of non-stationary signal from high frequency to low frequency. It is an adaptive decomposition. The process is as follow:

Search all the extremas of original signal $x(t)$. Calculate the mean value $m_{i}$ and envelope value $a_{i}$ by every two adjacent points:

$$
\begin{aligned}
& m_{i}=\left(n_{i}+n_{i+1}\right) / 2 \\
& a_{i}=\left|n_{i}-n_{i+1}\right| / 2
\end{aligned}
$$

Process the mean value $m_{i}$ and envelope value $a_{i}$ by moving average to obtain the local mean function $m_{11}(t)$ and envelope estimation function $a_{11}(t)$. Then, separate the local mean function $m_{11}(t)$ from the original signal $x(t)$ :

$$
h_{11}(t)=x(t)-m_{11}(t)
$$

Divide $h_{11}(t)$ by envelope estimation function $a_{11}(t)$ in order to demodulate the $h_{11}(t)$ :

$$
s_{11}(t)=h_{11}(t) / a_{11}(t)
$$

Repeat the process above, until $s_{1 n}(t)$ is a pure FM signal. $-1 \leq s_{1 n}(t) \leq 1$, its' envelope estimation function $a_{1 n+1}(t)$ satisfies $a_{1 n+1}(t) \approx 1$. Multiply all the envelope estimation function $a_{1 n}(t)$ during the iterative procedure to get the envelope signal $a_{1}(t)$ :

$$
a_{1}(t)=\prod_{n=1}^{N} a_{1 n}(t)
$$

Multiply the envelope signal $a_{1}(t)$ with pure FM function $s_{1 n}(t)$ to obtain the first PF:

$$
P F_{1}(t)=a_{1}(t) * s_{1 n}(t)
$$

Separate $P F_{1}(t)$ from original signal $x(t)$ to get a new signal $u_{1}(t)$. Repeat the process above, until the extremum of residual signal $u_{k}(t)$ is equal or less than 1 . The decomposition result of signal $x(t)$ is as follows:

$$
\begin{aligned}
& x(t)=\sum_{k=1}^{K} P F_{k}(t)+u_{k}(t) \\
& P F_{k}(t)=a_{k}(t) * s_{k}(t)
\end{aligned}
$$

\section{B. Identification method}

The free vibration system's differential equation is as follows:

$$
M \cdot \ddot{X}(t)+C \cdot \dot{X}(t)+K \cdot X(t)=0
$$

$\mathrm{M}, \mathrm{C}, \mathrm{K}$ means the mass matrix, stiffness matrix and damping matrix, $\mathrm{X}(\mathrm{t})$ is the response signal of the system.

Suppose $X(t)=U \cdot X_{N}(t)$, where $\mathrm{U}$ is a constant matrix, the equation (8) will be as follows:

$$
M \cdot U \cdot \ddot{X}_{N}(t)+C \cdot U \cdot \dot{X}_{N}(t)+K \cdot U \cdot X_{N}(t)=0
$$

Regularized modal matrixes meet the following conditions: 


$$
U^{T} \cdot M \cdot U=I
$$

$U^{T} \cdot K \cdot U=\left[\begin{array}{lll}\backslash & & \\ & \omega_{0 r}^{2} & \\ & & \backslash\end{array}\right]$

$U^{T} \cdot C \cdot U=\left[\begin{array}{lll}\backslash & & \\ & 2 \cdot \xi_{r} \cdot \omega_{0 r} & \\ & & \backslash\end{array}\right]$

Then the equation (9) can be expressed as follows:

$$
x_{N r}(t)+2 \cdot \xi_{r} \cdot \omega_{0 r} \cdot x_{N r}(t)+\omega_{0 r}^{2} \cdot x_{N r}(t)=0
$$

Where $r$ is the coordinate of the modal index. The solution of equation (13) is as follows:

$$
x_{N r}(t)=C_{r} \cdot e^{-\xi_{r} \cdot \omega_{o r} \cdot t} \cdot \sin \left(\omega_{d r} \cdot t+\varphi_{r}\right)(r=1,2, \cdots, n)
$$

In the equation (14), $\omega_{d r}=\omega_{0 r} \sqrt{1-\xi_{r}^{2}}, C_{r}$ and $\varphi_{r}$ are undetermined coefficients.

The viscous damping system vibration is the combination of every modal vibration [5]. The velocity instantaneous response is as follows:

$$
v(t)=\sum_{r=1}^{N} A_{r} \cdot n_{r} \cdot \omega_{d r} \cdot e^{-n_{r} \cdot t} \cdot \cos \left(\omega_{d r} \cdot t+\theta_{r}\right)
$$

In the equation (15), $n_{r}=c_{r} /\left(2 m_{r}\right), c_{r}$ is the damping of modal $\mathrm{r}, m_{r}$ is the mass of modal $\mathrm{r}$.

The instantaneous amplitude and instantaneous frequency of modal $r$ are as follows:

$$
A_{r}(t)=A_{r} \cdot n_{r} \cdot \omega_{d r} \cdot e^{-n_{r} \cdot t}, f_{r}(t)=\omega_{d r} / 2 \pi
$$

In the equation (16), the natural logarithms of the instantaneous amplitude are as follows:

$$
\ln A_{r}(t)=\ln \left(A_{r} \cdot n_{r} \cdot \omega_{d r}\right)-n_{r} \cdot t
$$

Where $n_{r}$ is the slope of fitting line of instantaneous amplitude's natural logarithm. Then the frequency-damping ratios of modal $r$ are calculated as equation (18):

$$
\xi_{r}=n_{r} / \omega_{d r}
$$

The parameter identification method based on LMD is showed in the following Fig.1: 


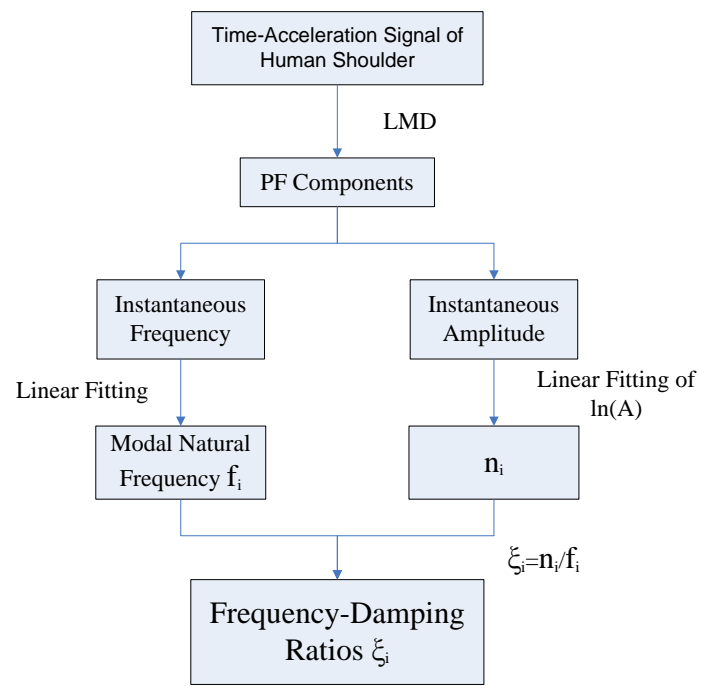

Fig. 1 The flowchart of parameter identification method based on LMD

\section{Identification Example}

The shooting test is carried out with high-speed photography. So the time-acceleration signal of human shoulder is gathered by calculating the movement of the mark point. The shooting conducts one round, and the cycle time is about $100 \mathrm{~ms}$. It is very short compare to the time of body movement. So the human body can be considered as free vibration.

The top view of shooting test is showed in Fig.2, where " 0 " is the mark point:

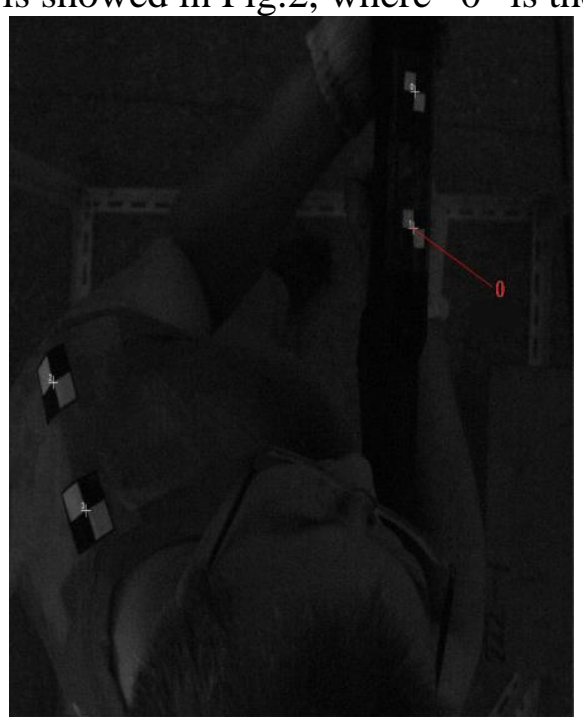

Fig. 2 Top view of shooting test

The time-acceleration signal of human shoulder is showed in Fig.3.

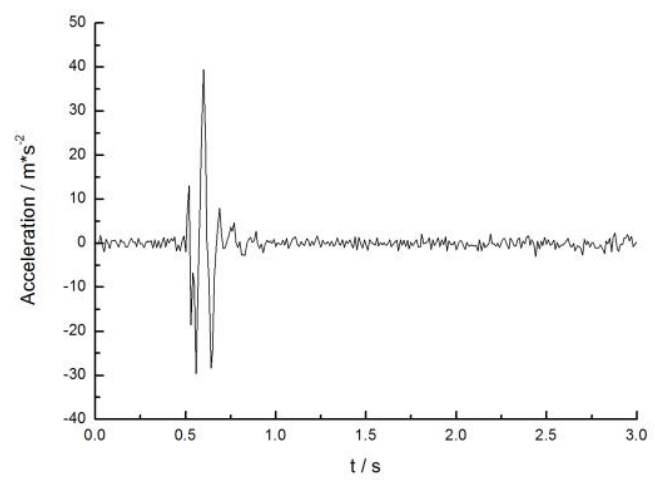

Fig. 3 Diagram of time-acceleration signal of human shoulder 
The LMD result of time-acceleration signal is showed in Fig.4, there are three natural frequencies of the human shoulder movement. The calculation results of $n_{i}$ and $f_{i}$ are showed in Fig.5, the full lines are the calculation results and the dashed lines are the liner fitting results. The spectrum of the calculation results should be liner theoretically, but they in fact have some small perturbations. That is because: 1) free vibration hypothesis; 2) riding waves in LMD; 3) noise interference. Tab.1 is the parameter identification results, and the results in reference [3] are also presented. It shows that the identification results have the congruent relationship with reference [3], and they are credible.
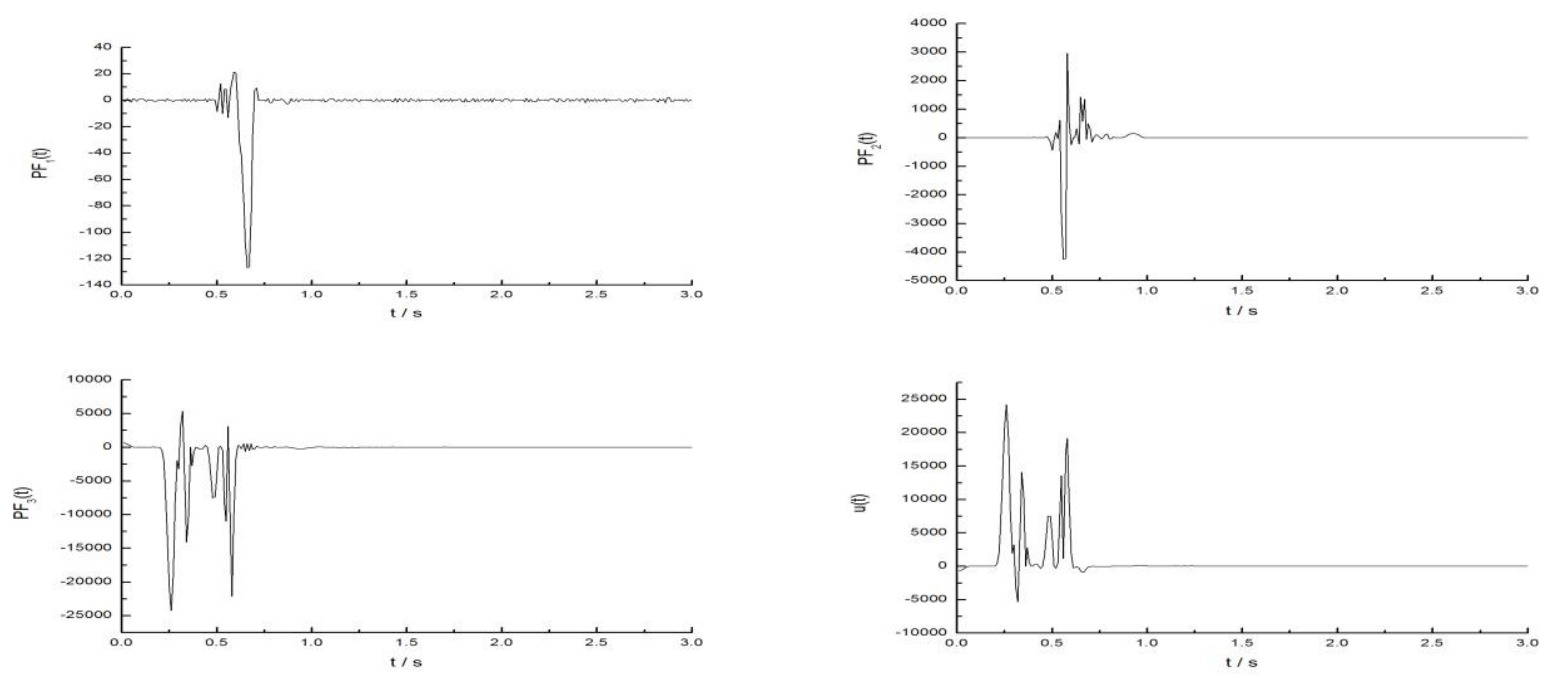

Fig. 4 LMD result of time-acceleration signal
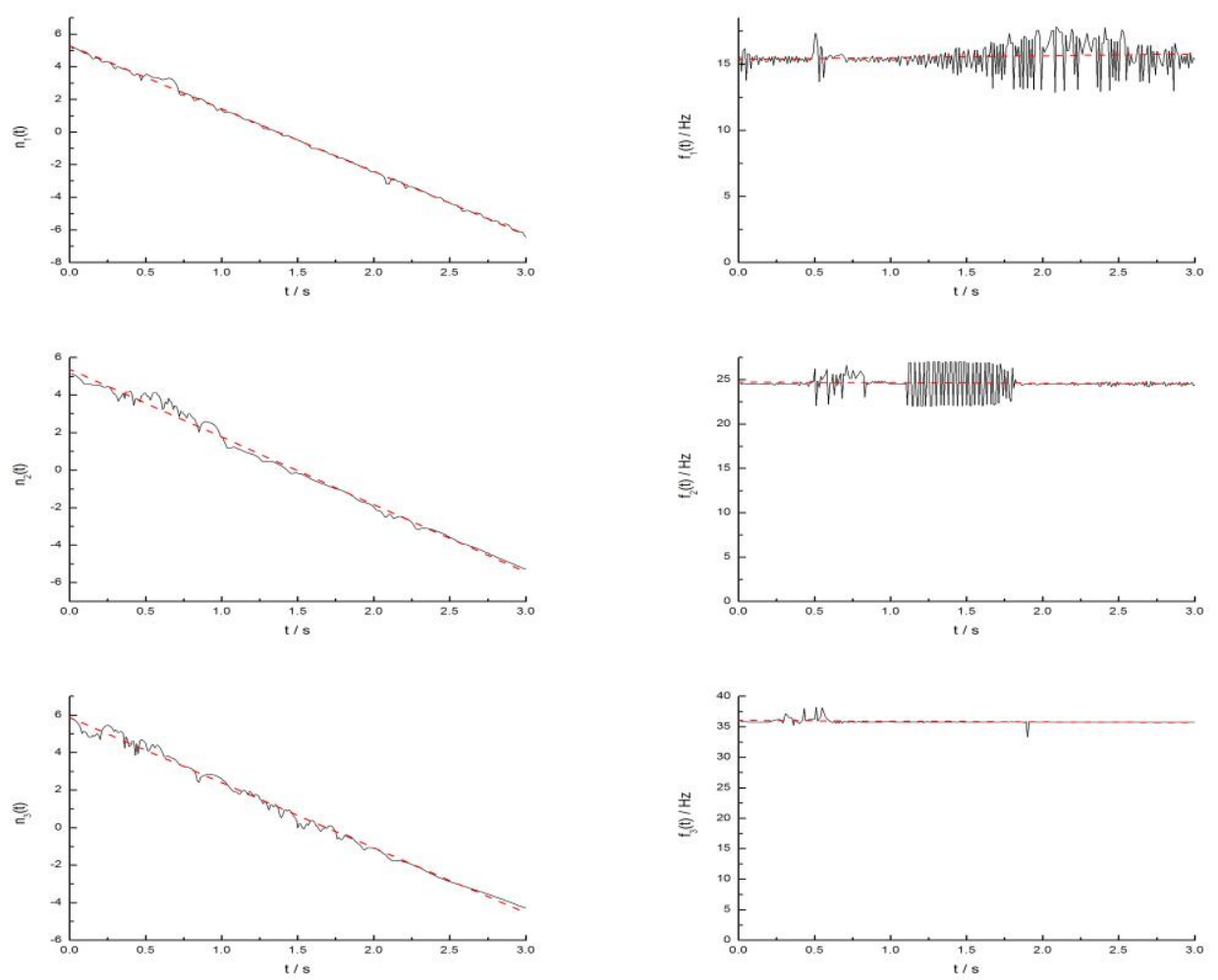

Fig.5 Diagram of calculation results $n_{i}$ and $f_{i}$ 
Tab.1 Parameter identification results

\begin{tabular}{ccccccc}
\hline \multirow{2}{*}{1} & \multicolumn{2}{c}{ Modal 1 } & \multicolumn{2}{c}{ Modal 2 } & \multicolumn{2}{c}{ Modal 3 } \\
\cline { 2 - 7 } & $f_{1} / \mathrm{Hz}$ & $\xi_{1}$ & $f_{2} / \mathrm{Hz}$ & $\xi_{2}$ & $f_{3} / \mathrm{Hz}$ & $\xi_{3}$ \\
\hline $\begin{array}{c}\text { LMD } \\
\text { method }\end{array}$ & 15.35 & 0.249 & 24.53 & 0.143 & 35.79 & 0.089 \\
$\begin{array}{c}\text { Reference } \\
{[3]}\end{array}$ & 15.06 & 0.216 & 24.12 & 0.158 & 35.19 & 0.096 \\
\hline
\end{tabular}

\section{Summary}

In this paper, the physical parameters of human body in passive state are identified based on LMD. The parameter identification result shows two conclusions:

1) The parameter identification is credible, and it is more effective and programmable than the method in reference [3]. Therefore, it is of great importance for the study of human biomechanics.

2) There are 3 nature frequencies of human shoulder movement. That means the human shoulder's movement can be simulated with a dynamic model of 3 degrees of freedom. They are displacement, pitch and rotate.

\section{References}

[1] Ding YuLan. Ergonomics[M] Beijing University of Technology Press.2002.2

[2] Liu Guangliao. Automatic Weapon Measuring and Testing Technique[M] Nanjing University of Science and technology,1986.6.

[3] Kong Deren, Sun Haibo, Li Yongxin, Zhu Minwu, Liu Guangliao. Mechanical Admittance Analysis of Rifle-shoulder System[J]. Journal of Nanjing University of Science and Technology,1997, 21(5):441-444

[4] ZHU Wenfeng. Research On Modal Parameters Identification Based On Local Mean Decomposition Method[D]. Hunan University,2014,1-69.

[5] CHENG Junsheng, SHI Meili, YANG Yu. Roller bearing fault diagnosis method based on LMD and neural network[J]. Journal of Vibration and Shock,29(8):141-144 